\title{
Limited Efficacy of Calcium and Magnesium in a Porcine Model of Hydrofluoric Acid Ingestion
}

\author{
Jason A. Coffey, MD', Kori L. Brewer, PhD ${ }^{a}$, Robert Carroll, PhD ${ }^{b}$, John Bradfield, DVM, PhD', \\ William J. Meggs, MDa
}

aDepartment of Emergency Medicine, Brody School of Medicine at East Carolina University, Greenville, NC

bDepartment of Physiology, Brody School of Medicine at East Carolina University, Greenville, NC

cDepartment of Comparative Medicine, Brody School of Medicine at East Carolina University, Greenville, NC

\begin{abstract}
Objective: This investigation evaluated the effectiveness of calcium and magnesium in treating oral hydrofluoric acid (HF) poisoning.

Methods: The controlled laboratory investigation used anesthetized pigs. Subjects received HF via NG tube, titrated to abolish electrocardiographic abnormalities. The untreated group received saline infusion. The treatment group received serial injections of calcium chloride $\left(\mathrm{CaCl}_{2}\right)$ and magnesium chloride $\left(\mathrm{MgCl}_{2}\right)$. A third group received oral infusions of Calcium fluoride $(\mathrm{CaF} 2)$. We measured heart rate, QRS interval, $\mathrm{pH}$, bicarbonate, calcium, magnesium, and potassium. The Wilcoxon Rank Sum test was used to compare intra- and inter-subject differences.

Results: Fatality occurred in all pigs receiving HF. Compared to the untreated group, trends for the treatment group were toward a larger amount of HF to produce fatality $(83.1 \pm 17.5$ grams vs. $37.7 \pm 16.1$ grams, $\mathrm{p}=0.08)$, to cause QRS prolongation (72.5 \pm 25.8 vs. $33.8 \pm 14.9$ grams, $\mathrm{p}=0.08)$, and to lower potassium at mortality ( $4.9 \pm 0.7 \mathrm{vs.} 8.7 \pm 2.7 \mathrm{mEq} / \mathrm{L}, \mathrm{p}=0.08)$. No major changes in calcium $(-1.0 \pm 0.7 \mathrm{mEq} / \mathrm{L})$ or magnesium $(0.4 \pm 0.6 \mathrm{mEq} / \mathrm{L})$ occurred in the untreated group. Tachycardia developed in all pigs and ventricular arrhythmias occurred in 2 of 3 pigs of both groups [ $\mathrm{CaF}_{2}$ administration caused no QRS prolongation or ventricular arrhythmias and had no effect on laboratory parameters].

Conclusion: $\mathrm{CaCl}_{2}$ and $\mathrm{MgCl}_{2}$ replacement delayed but did not prevent fatality and QRS prolongation. Although this result suggests $\mathrm{Ca}++$ and $\mathrm{Mg}++$ may be beneficial in the treatment of systemic HF toxicity, factors other than hypocalcemia and hypomagnesemia play a role in toxicity.
\end{abstract}

\section{INTRODUCTION}

HF is available in industry and in household products, and it ranges in concentrations from $3 \%$ to $70 \%$. HF is used for glass etching, brick cleaning, etching chips in the semiconductor industry, electroplating, leather tanning, rust removal, and the cleaning of porcelain [1]. Even though it is not completely understood, systemic toxicity from HF is associated with a high incidence of mortality. Chelation of divalent cations is one of the theories regarding toxicity, especially when $\mathrm{Ca}^{++}$is the mechanism. HF may produce life-threatening systemic toxicity with large or highly concentrated dermal exposures, with inhalation, and with

Keywords: hydrofluoric acid, poisoning, cardiac arrhythmias, magnesium, calcium Notes: There was no outside funding of any kind used for this study.

Acknowledgements: The authors wish to thank Christopher S. Coffey, PhD, of the Department of Biostatistics, University of Alabama at Birmingham, Birmingham, Alabama, for his help with the statistical analysis.

Corresponding Author: William J. Meggs, MD, PhD, Room 4W54, Brody School of Medicine at East Carolina University, Greenville, NC 27858. Email: meggsw@mail.ecu.edu 
ingestion. The current treatment of HF exposure includes decontamination, replacement of calcium and magnesium, and topical or intradermal calcium at local burn sites [1].

A previous study found that ingestion of household HF is not uncommon and may lead to sudden death without warning signs. A number of case reports have described instances where oral ingestion of a HF solution resulted in cardiac arrhythymias or death [8-14]. The effectiveness of intravenous (IV) calcium and magnesium replacement in systemic HF toxicity has been evaluated in case reports, but it has never been studied in an animal model. Several case reports describe ventricular dysrhythmias or death, despite replacement of calcium and magnesium $[10-12,17]$. These cases question the effectiveness of IV calcium and magnesium in the treatment of systemic HF poisoning, and they suggest the possibility of something other than hypocalcemia or hypomagnesemia as the cause of ventricular arrhythmias.

This study uses a porcine model to determine the effectiveness of IV calcium and magnesium in preventing electrocardiographic abnormalities associated with systemic HF poisoning. We also investigated the toxicity of oral $\mathrm{CaF}$. The null hypothesis investigated was that IV calcium and magnesium would not prevent asystole in systemic HF poisoning.

\section{MATERIALS AND METHODS}

The animal care and use committee of the Brody School of Medicine at East Carolina University approved this investigation. Protective equipment and safety procedures were approved through the university's Department of Environmental Health and Safety. While handling the HF, strict safety precautions were coordinated through the university's Department of Environmental Health and Safety. We obtained $48 \% \mathrm{HF}$ solution, $\mathrm{CaCl}_{2}$, and $\mathrm{MgCl}_{2}$ from Sigma-Aldridge Chemical Corporation (Allentown, PA), and we obtained adolescent pigs with a body weight ranging from 19.4-22 kilograms from the School of Veterinary Medicine, North Carolina State University. Serum electrolyte concentration of calcium $\left(\left[\mathrm{Ca}^{2+}\right]\right)$, magnesium $\left(\left[\mathrm{Mg}^{2+}\right]\right)$, and potassium $\left(\left[\mathrm{K}^{+}\right]\right)$concentrations and arterial blood gases were measured at the clinical laboratory of the animal care center. This certified laboratory employs trained and licensed laboratory technicians. Plasma fluoride $\left(\left[\mathrm{F}^{-}\right]\right.$) levels were measured at the American Medical Laboratories (Chantilly, VA).

A controlled prospective laboratory investigation was undertaken using anesthetized pigs. Pigs were fasted on the evening prior to the experiments. At the beginning of each experiment, pigs were sedated with intramuscular (IM) Telazol, $5 \mathrm{mg} / \mathrm{kg}$, and Xylaxine, $1 \mathrm{mg} / \mathrm{kg}$, prior to endotracheal intubations. Anesthesia was maintained with a 2:1 nitrous oxide to oxygen mixture and 1-2\% Isoflourane, titrated to maintain general anesthesia. NG tube placement was confirmed by return of gastrointestinal contents and by auscultation of air. At the conclusion of the experiments, the pigs were euthanized with IV sodium pentobarbital $(100 \mathrm{mg} / \mathrm{kg})$.
After induction of general anesthesia, the marginal ear vein was cannulated and $0.9 \%$ normal saline was infused at $60 \mathrm{ml} / \mathrm{hr}$. A femoral arterial line was established by surgical dissection and used for blood pressure measurements and blood collections. One $\mathrm{mL}$ samples of arterial blood were collected for arterial blood gas analyses and measurement of $\mathrm{K}, \mathrm{Mg}$, and $\mathrm{Ca}$ concentrations. Cardiac rhythm and blood pressure were continuously monitored. QRS interval was measured on the rhythm strip as the distance from the beginning of the $\mathrm{Q}$ wave to the end of the $\mathrm{S}$ wave. We defined QRS prolongation as an increase in the QRS interval from the baseline.

We based HF dose on case reports of HF oral exposure [8-10, $12,13,15,22]$. We estimated that a 3 ounce solution of $6 \%$ HF was approximate to $\mathrm{LD}_{50}$ in a $70 \mathrm{~kg}$ human. Assuming a similar absorption and systemic response to HF as humans, a dose of $0.13 \mathrm{ml} / \mathrm{kg}$ of $48 \%$ HF solution was the estimated $\mathrm{LD}_{50}$ in a pig. The HF dose was administered by NG tube and repeated every 5 minutes until the onset of electrocardiographic abnormalities. $\mathrm{CaCl}_{2}$ and $\mathrm{MgCl}_{2}$ were intravenously given through a previously established catheter in the marginal ear vein of each pig. To obtain comparable and standard pharmacy dosing, $\mathrm{CaCl}_{2}$ was mixed as $1 \mathrm{gm} / 20 \mathrm{ml}$ of $0.9 \%$ normal saline, and $\mathrm{MgCl}_{2}$ was mixed as $1 \mathrm{gm} / 25 \mathrm{ml}$ of $0.9 \%$ normal saline.

In Group 1 (the untreated group), 3 pigs received $0.13 \mathrm{~mL} / \mathrm{kg}$ of a $48 \%$ HF solution, diluted 1:3 with tap water and administered through an NG tube followed by $15 \mathrm{~mL}$ of tap water. This dose was repeated every 5 minutes until the clinical endpoint (cardiac asystole) was achieved.

In Group 2 (the treatment group), 3 pigs, following the above protocol, received HF. Each dose of HF was followed by an IV dose of $\mathrm{CaCl}_{2}(0.10 \mathrm{ml} / \mathrm{kg}$ of the mixed solution or $5 \mathrm{mg} / \mathrm{kg}$ per dose $)$ and $\mathrm{MgCl}_{2}(0.26 \mathrm{~mL} / \mathrm{kg}$ of the mixed solution or $10.5 \mathrm{mg} / \mathrm{kg}$ per dose). We used data from pilot studies and molar calculations to estimate the dose of $\mathrm{CaCl}_{2}$ and $\mathrm{MgCl}_{2}$ needed to keep each pig's calcium and magnesium level within the normal range. Again, the clinical endpoint was defined as cardiac asystole.

We compared the mean dose of HF that caused fatality and QRS prolongation of pigs in Group 1 and Group 2. The mean serum concentrations of potassium, magnesium, and calcium prior to mortality as well as the mean change in potassium, magnesium, calcium, $\mathrm{pH}$, and bicarbonate from baseline to mortality was also compared in pigs from Group 1 and Group 2. Results are reported as mean values with standard deviation. Due to small sample size, a Wilcoxon Rank sum test was used to compare the ranking of observations between the 2 groups. For analysis, a $\mathrm{p} \leq 0.05$ was considered significant.

\section{RESULTS}

The result of each comparison is summarized in Table 1. The administration of HF in both groups was fatal. Asystole occurred in all 3 pigs in Group 1 and all 3 pigs in Group 2. Also, QRS prolongation occurred in all pigs in each group: Group 1 experienced asystole 9-15 minutes after QRS prolongation, and Group 2 experienced asystole 3-61 minutes after QRS prolongation. 
Table 1: Outcomes for comparison among Group 1 and Group 2 subjects

\begin{tabular}{lccc}
\hline & Group 1 $(\mathbf{n}=\mathbf{3})$ & Group 2 (n=3) & p-value \\
\hline HF dose to fatality (grams) & $37.7 \pm 16.1$ & $83.1 \pm 17.5$ & 0.08 \\
\hline HF dose to QRS prolongation (grams) & $33.8 \pm 14.9$ & $72.5 \pm 25.8$ & 0.19 \\
\hline Potassium (mEq/L) at mortality & $8.7 \pm 2.7$ & $4.9 \pm 0.7$ & 0.08 \\
\hline Change in Potassium (mEq/L) & $5.0 \pm 2.7$ & $1.6 \pm 0.6$ & 0.19 \\
\hline Magnesium (mEq/L) at mortality & $1.8 \pm 0.5$ & $6.3 \pm 3.7$ & 0.08 \\
\hline Change in Magnesium (mEq/L) & $0.4 \pm 0.6$ & $4.5 \pm 3.3$ & 0.08 \\
\hline Calcium (mg/dL) at mortality & $7.3 \pm 0.4$ & $13.4 \pm 1.8$ & 0.08 \\
\hline Change in Calcium (mg/dL) & $-1.0 \pm 0.7$ & $4.5 \pm 1.5$ & 0.08 \\
\hline Change in pH & $-0.171 \pm 0.214$ & $-0.192 \pm 0.032$ & 0.66 \\
\hline Change in Bicarbonate (mmol/L) & $-11.3 \pm 0.4$ & $-7.5 \pm 5.0$ & 0.66 \\
\hline
\end{tabular}

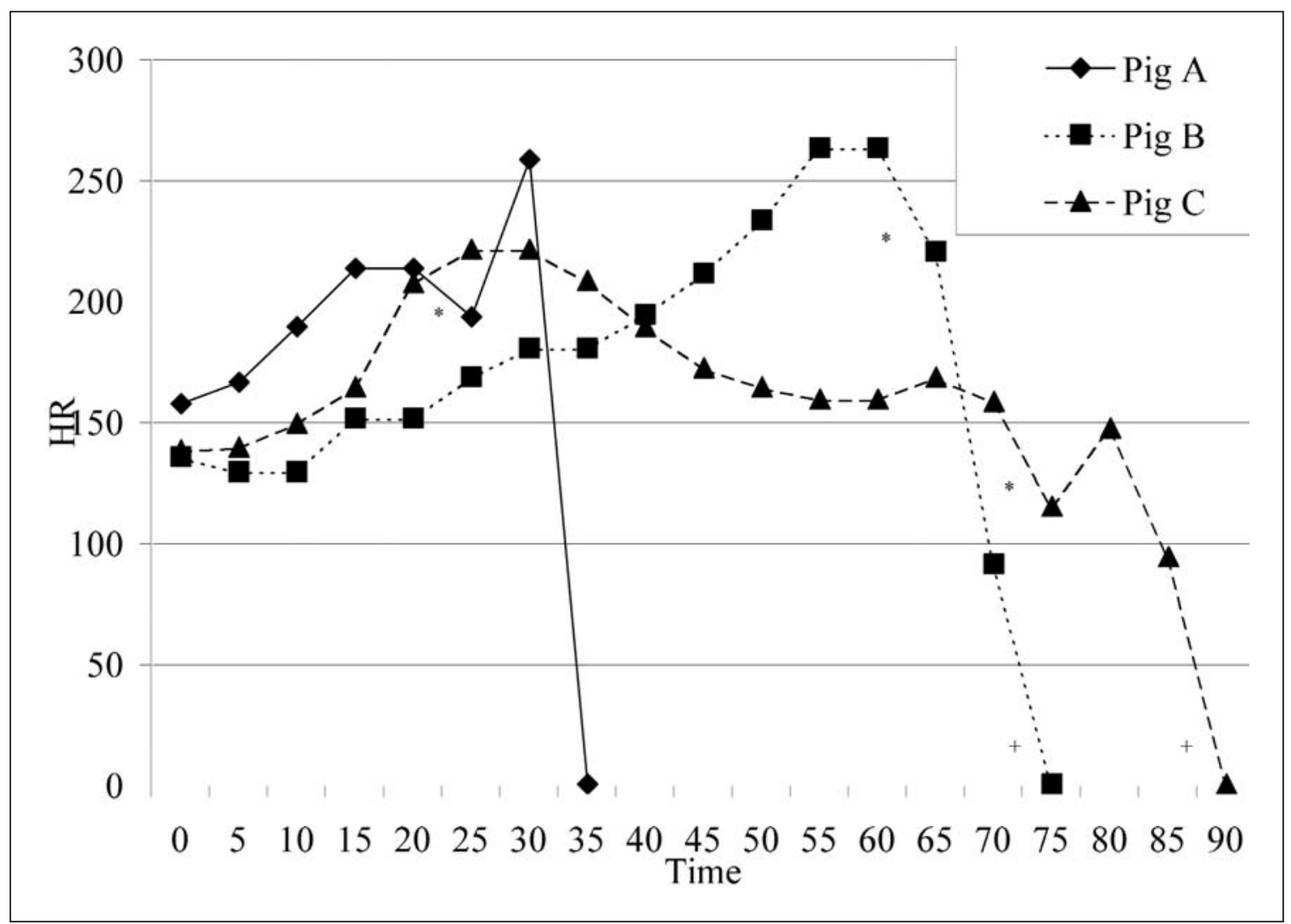

Figure 1: Effect of hydrofluoric acid administration on heart rate in Group 1 pigs.

The pigs in Group 1 showed a trend toward higher serum potassium concentration prior to mortality then did the pigs in Group 2. As expected, $\mathrm{Ca}++$ and $\mathrm{Mg}++$ levels prior to mortality were higher in Group 2 since calcium and magnesium were replaced. Group 1 and Group 2 did not show any statistical significance between the mean calcium or magnesium levels prior to mortality, and they did not show a change in calcium or magnesium. Also, the change in $\mathrm{pH}$ and bicarbonate was not significantly different between the 2 groups.

Tachycardia developed in both groups (Figures 1 \& 2). The maximum heart rate of pigs in Group 1 was $247 \pm 23$, and the maximum heart rate of pigs in Group 2 was $201 \pm 29$. The dose of HF that produced maximum heart rate for Group 1 and Group 2 were $24.2 \pm 9.3$ and $61.9 \pm 23.3$ respectively. In Group 1, 


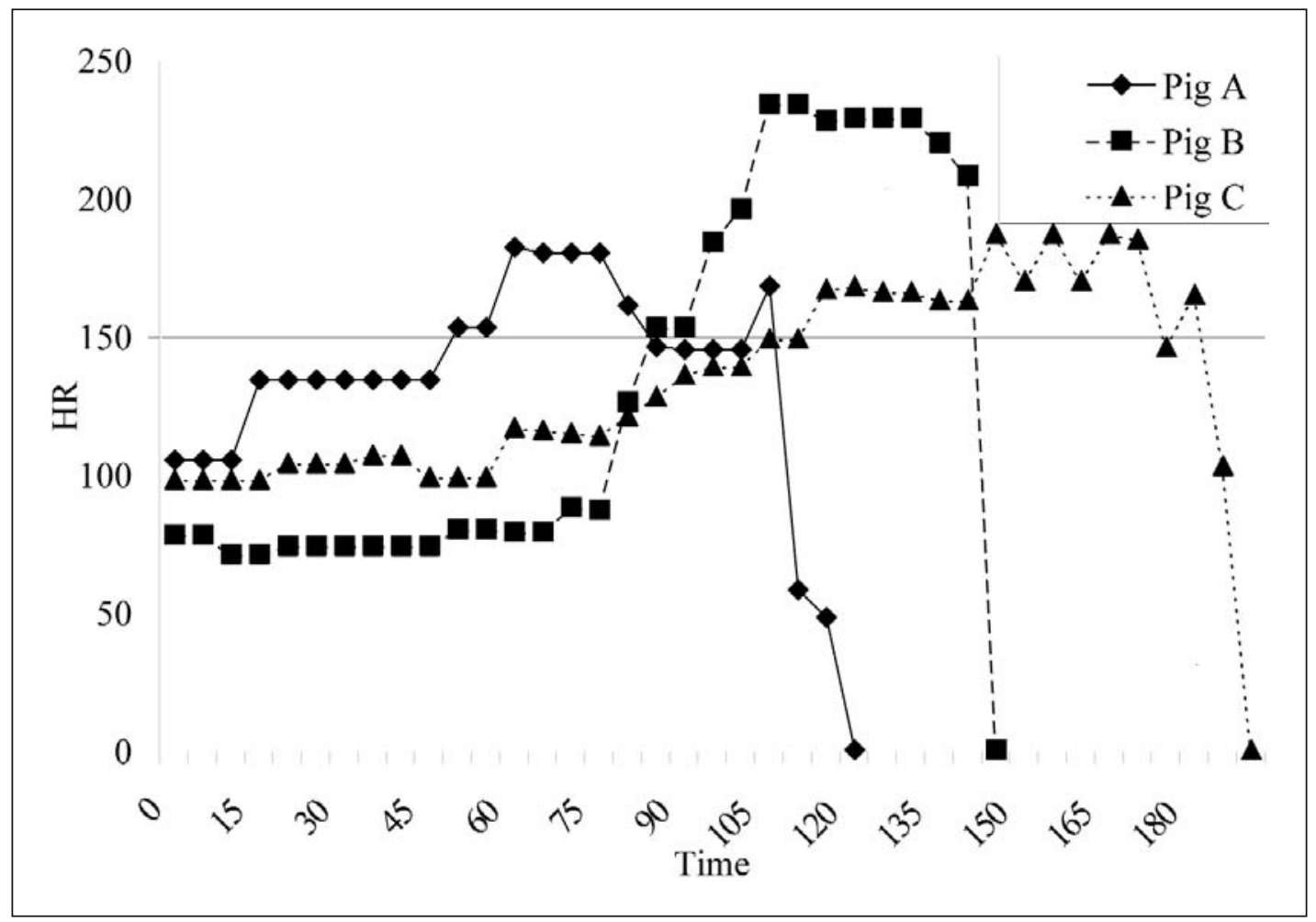

Figure 2: Effect of hydrofluoric acid administration on heart rate in Group 2 pigs.

ventricular tachycardia/fibrillation occurred in pig B, and ventricular tachycardia occurred in pig C prior to asystole (Figure 1). In Group 2, torsade de pointes/ventricular tachycardia occurred in pig B and torsade de pointes in pig C prior to asystole (Figure 2). The onset of ventricular arrhythmias occurred 1 minute prior to asystole in Group 1 pigs and occurred 0 and 2 minutes prior to asystole in Group 2 pigs.

Plasma fluoride levels were not obtained from the Group 1 pigs. We obtained fluoride levels of $15.2,62$, and $76(\mathrm{mg} / \mathrm{L})$ from pigs $\mathrm{A}, \mathrm{B}$, and $\mathrm{C}$ of Group 2 . These levels were markedly elevated in comparison to normal laboratory ranges $(0.2-0.8 \mathrm{mg} / \mathrm{L})$. The higher plasma levels of fluoride were seen in the pigs that required more $\mathrm{HF}$ to cause mortality $(66.7,81.2$, and 101.5 grams among pigs $\mathrm{A}, \mathrm{B}$, and $\mathrm{C}$ respectively).

All three pigs in Group 3 survived until termination of the experiment. A mild tachycardia was seen among all three pigs with a mean maximum heart rate of $141 \pm 19$ (Figure 3). Tachycardia resolved in pigs A \& B prior to the end of the study. Tachycardia was persistent in pig $\mathrm{C}$ upon termination of the study. The mean change in heart rate from baseline was $37 \pm 30$. Among the 3 pigs, there was no significant change in the potassium $(0.3 \pm 0.3 \mathrm{mEq} / \mathrm{L})$, calcium $(-0.7 \pm 0.3 \mathrm{mg} / \mathrm{dL})$, magnesium $(0.1 \pm 0.3 \mathrm{mEq} / \mathrm{L}), \mathrm{pH}(0.015 \pm 0.019)$, or bicarbonate $(-0.7 \pm 0.2$ $\mathrm{mmol} / \mathrm{L}$ ) from baseline to termination of the study. No QRS prolongation or arrhythmias developed. We obtained plasma fluoride levels of all 3 pigs prior to termination of the study. The plasma fluoride levels were slightly elevated at $0.19,0.13$, and $0.22 \mathrm{mg} / \mathrm{L}$ respectively.

\section{DISCUSSION}

Using a pig model, we demonstrated a trend that suggests calcium and magnesium have a protective effect against the systemic effects of HF. Although these differences did not reach statistical significance, the pigs that received calcium and magnesium lived longer and required larger amounts of HF to produce QRS prolongation and fatality when compared to the untreated group. All pigs died, however, indicating that there may be some factor other than hypocalcemia or hypomagnesemia involved in deaths related to systemic HF toxicity. The fluoride ion may play a role in the lethality that is seen. Plasma fluoride levels were not available for Group 1 pigs, but they were markedly elevated in the Group 2 pigs. Plasma fluoride levels at fatality (reported in the literature) ranged from $8-56.2 \mathrm{mg} / \mathrm{L}$ $[9,13,15]$. Our pigs achieved levels of $15.2-76$, and these levels are consistent with the range found in the human reported fatalities.

There are several theories accounting for cardiac deaths related to systemic HF poisoning. The most commonly accepted theory is that fluoride binds to calcium and magnesium forming insoluble salts, thus depleting calcium and magnesium supplies and causing arrhythmias. Although one would expect a drop in 


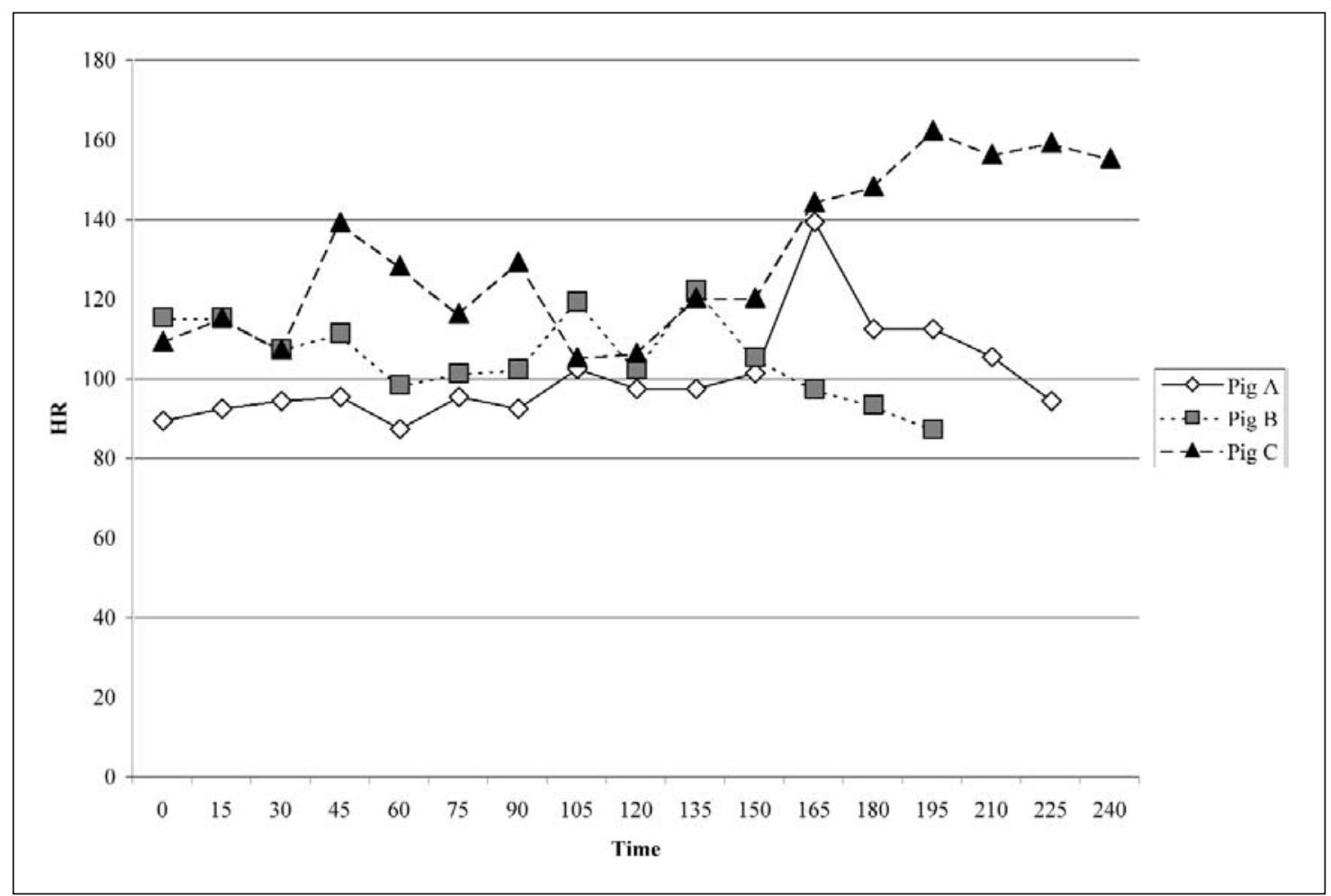

Figure 3: Effect of calcium fluoride administration on heart rate in Group 3 pigs plotted over time.

calcium and magnesium for the untreated group that received a substantial amount of HF, our study conversely showed only a minor drop in calcium $(-1.0 \pm 0.7 \mathrm{mEq} / \mathrm{L})$ and no major change in magnesium $(0.4 \pm 0.6 \mathrm{mEq} / \mathrm{L})$. The treated pigs developed ventricular arrhythmias and death with slightly elevated serum calcium and magnesium levels.

Potassium efflux from calcium dependent potassium channels has been associated with ventricular arrhythmias and death in animal models of systemic fluoride poisoning [23,24]. In an animal model, hyperkalemia has been more closely associated with EKG changes and ventricular arrhythmias than hypocalemia $[25,26]$. In our untreated pig group, hyperkalemia was present prior to mortality in all 3 pigs. Hyperkalemia, however, did not develop in the pigs treated with calcium and magnesium. Group 2 pigs had QRS widening and developed ventricular arrhythmias similar to Group 1 pigs, and thus it is difficult to make a direct correlation between potassium and cardiac toxicity in our model. Calcium and magnesium may have a protective effect against the development of hyperkalemia in systemic poisoning.

A consistent finding in the literature regarding systemic HF poisoning is precipitous death or ventricular arrhythmias without warning signs. In our animal model, all 3 pigs of each group developed tachycardia relatively early. QRS prolongation, however, was a late finding and was seen between 3 and 61 minutes prior to asystole in the 2 groups. Based on our study, QRS prolongation would not be an adequate way to predict toxicity because of the delayed finding. Our study suggests that tachycardia, though a non-specific finding, may be an early predictor of cardiac toxicity.

\section{LIMITATIONS}

There are several limitations of this study that should be mentioned. First of all, the study groups were limited to a few pigs. We were able to show only trends toward increased HF dose to fatality, less hyperkalemia, and delayed QRS prolongation among the group treated with calcium and magnesium compared to the untreated group. If the study groups had a greater number of pigs, these differences would likely have been statistically significant. A second limitation is that we did not pursue any therapeutic interventions commonly done in clinical practice. In our study, we wanted to evaluate the effect of systemic HF toxicity on $\mathrm{pH}$ and bicarbonate in a treated and untreated group and thus no corrections were made to maintain a normal acid-base status. We did not attempt to reverse ventricular arrhythmias with chemicals or electricity and thus cannot make a statement on whether ventricular arrhythmias are more prone to conversion in the presence of calcium and magnesium. Correction of the acid-base status and defibrillation attempts may have made this study more relevant in application to clinical practice.

Another limitation is that plasma fluoride levels were not obtained for the Group 1 pigs. Thus, the effect of calcium and magnesium on fluoride absorption cannot be evaluated. Worth noting is that we over-corrected magnesium and calcium in the 
Group 2 pigs and so causing hypermagnesemia $(6.3 \pm 3.7 \mathrm{mEq} / \mathrm{L})$ and hypercalcemia $(13.4 \pm 1.8 \mathrm{mEq} / \mathrm{L})$. We believe these levels were probably not high enough to produce the observed cardiac effects.

In summary, the administration of intravenous calcium and magnesium in the HF poisoned subject resulted in a trend toward delay in fatality, delay in QRS prolongation, and less hyperkalemia when compared to untreated subjects. Thus, calcium and magnesium administration may have a limited cardioprotective effect in systemic poisoning. Even with the administration of calcium and magnesium, systemic HF poisoning caused death in all subjects. Most of these deaths were related to ventricular arrhythmias. The universal fatality (even those with calcium and magnesium treatment) suggests that another factor may play a role in the ventricular arrhythmias and death seen with systemic toxicity. Our study suggests that this factor is something other than hypocalcemia and hypomagnesemia. This other factor may be the fluoride ion itself, acidosis, calcium fluoride, or some other unknown variable. Our study is inconclusive on the question of calcium fluoride as a potential cardiac irritant and further study may be indicated to pursue the calcium fluoride question. Like previous reports, onset of ventricular arrhythmias and death among the subjects was often rapid and occurred soon after onset of any QRS prolongation. Tachycardia, however, was seen among all of the subjects and may be a useful predictor of patients at risk for systemic toxicity.

Calcium and magnesium administration were associated with a delay in the onset of cardiac abnormalities, and (with other supportive care and aggressive interventions for cardiac arrhythmias) may prevent the almost universal fatality seen with severe systemic toxicity. From this study, administration of calcium and magnesium were not effective in preventing death from systemic hydrofluoric acid poisoning. The mechanism of cardiac toxicity in fluoride poisoning, likewise, remains poorly understood. To discover better therapeutic interventions, further investigation is needed to determine the exact cause of cardiac toxicity with systemic hydrofluoric acid poisoning.

The authors have no potential financial conflicts of interest to report.

\section{REFERENCES}

1. Fulton JA, Rao RB. Hydrofluoric acid and fluorides. In: Flomenbaum NE et al., Eds. Goldfrank's Toxicologic Emergencies, $8^{\text {th }}$ Edition. McGraw-Hill. New York. 2006.

2. Seyb ST, Noordhoek L, Botens S, Mani MM. A study to determine the efficacy of treatments for hydrofluoric acid burns. Journal of Burn Care \& Rehabilitation. 1995;16(3):253-237.

3. Williams JM, Hammad A, Cottington EC, Harchelroad FC. Intravenous magnesium in the treatment of hydrofluoric acid burns in rats. Annals of Emergency Medicine. 1994;23(3):464-469.

4. Dowbak G, Rose K, Rohrich RJ. A biochemical and histologic rationale for the treatment of hydrofluoric acid burns with calcium gluconate. Journal of Burn Care \& Rehabilitation.

1994; 15(4):323-327.

5. Burkhart KK, Brent J, Kirk MA, Baker DC, Kulig KW. Comparison of topical magnesium and calcium treatment for dermal hydrofluoric acid burns. Annals of Emergency Medicine. 1994;24(1):9-13.

6. Cox RD, Osgood KA. Evaluation of intravenous magnesium sulfate for the treatment of hydrofluoric acid burns. Journal of Toxicology-Clinical Toxicology. 1994;32(2):123-136.

7. Siegel DC, Heard JM. Intra-arterial calcium infusion for hydrofluoric acid burns. Aviation Space \& Environmental Medicine. 1992;63(3):206-211.

8. Kao WF, Dart RC, Kuffner E, Bogdan G. Ingestion of lowconcentration hydrofluoric acid: an insidious and potentially fatal poisoning. Annals of Emergency Medicine. 1999;34(1):35-41.

9. Bost RO, Springfield A. Fatal hydrofluoric acid ingestion: a suicide case report. Journal of Analytical Toxicology. 1995;19(6): $535-536$.

10. Stremski ES, Grande GA, Ling LJ. Survival following hydrofluoric acid ingestion. Annals of Emergency Medicine. 1992; 21(11):1396-1399.

11. Chan BS, Duggin GG. Survival after a massive hydrofluoric acid ingestion. Journal of Toxicology-Clinical Toxicology. 1997; 35(3):307-309.

12. Manoguerra AS, NeumanTS. Fatal Poisoning from hydrofluoric acid ingestion. American Journal of Emergency Medicine.

1986;4(4):362-363.

13. Menchel SM, Dunn WA. Hydrofluoric acid poisoning. American Journal of Forensic Medicine \& Pathology. 1984; 5(3):245-248.

14. Curry AS. Twenty-one uncommon cases of poisoning. British Journal of Medicine. 1962;1:687-689.

15. Bordelon BM, Saffle JR, Morris SE. Systemic fluoride toxicity in a child with hydrofluoric acid burns: case report. Journal of Trauma-Injury Infection \& Critical Care. 1993;34(3):437-439.

16. Yamaura K, Kao B, Iimori E, Urakami H, Takahashi S. Recurrent ventricular tachyarrhythmias associated with QT prolongation following hydrofluoric acid burns. Journal of Toxicology-Clinical Toxicology. 1997;35(3):311-313.

17. Greco RJ, Hartford CE, Haith LR Jr., Patton ML. Hydrofluoric acid-induced hypocalcemia. Journal of TraumaInjury Infection \& Critical Care. 1988;28(11):1593-1596.

18. Greendyke RM, Hodge HC. Accidental death due to hydrofluoric acid. Journal of Forensic Science. 1964;9:383.

19. Mayer TG, Gross PL. Fatal systemic fluorosis due to hydrofluoric acid burns. Annals of Emergency Medicine. 1985;14:149.

20. Mullett T, Zoeller T, Bingham H, et al. Fatal hydrofluoric acid cutaneous exposure with refractory ventricular fibrillation. Journal of Burn Care \& Rehabilitation. 1987;8:216.

21. Tepperman PB. Fatality due to acute systemic fluoride poisoning following a hydrofluoric acid skin burn. Journal of Occupational Medicine. 1980;22:691.

22. Klasner AE, Scalzo AJ, Blume C, Johnson P, Thompson MW. Marked hypocalcemia and ventricular fibrillation in two 
pediatric patients exposed to a fluoride-containing wheel cleaner. Annals of Emergency Medicine. 1996;28(6):713-718.

23. Cummings CC, McIvor ME. Fluoride-induced hyperkalemia: the role of the $\mathrm{Ca}^{+}{ }^{+}$-dependent $\mathrm{K}^{+}$channels. American Journal of Emergency Medicine. 1988;6(1):1-3.

24. McIvor ME, Cumming CC. Sodium fluoride produces a $\mathrm{K}^{+}$ efflux by increasing intracellular $\mathrm{Ca} 2^{+}$through $\mathrm{Na}+\mathrm{Ca} 2+$ exchange. Toxicology Letters. 1987;38(1-2):169-176.
25. McIvor ME, Cumming CC, Mower MM, et al. Sudden cardiac death from acute fluoride intoxication: the role of potassium. Annals of Emergency Medicine. 1987;16(7):

777-781.

26. McIvor ME, Cummings CC, Mower MM, et al. The manipulation of potassium efflux during fluoride intoxication: implications for therapy. Toxicology. 1985; 37(3-4):233-239. 